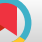

\title{
Dentists' Knowledge and Practice of the Notification Protocol of the Ministry of Health in Dealing with COVID-19: A Cross-sectional Study in Khuzestan
}

\author{
Mohammad Shooriabi (iD ${ }^{1}$ and Sedigheh Modarres Mousavy (iD) ${ }^{1, *}$ \\ ${ }^{1}$ Department of Oral Medicine, Faculty of Dentistry, Ahvaz Jundishapur University of Medical Sciences, Ahvaz, Iran \\ "Corresponding author: Department of Oral Medicine, Faculty of Dentistry, Ahvaz Jundishapur University of Medical Sciences, Ahvaz, Iran. Email: fmousavy@gmail.com
}

Received 2020 December 30; Revised 2021 September 21; Accepted 2021 September 21.

\begin{abstract}
Background: The number of patients suffering coronavirus (COVID-19) is increasing worldwide, and given that there is now no vaccine against this virus, the best prevention technique is to observe health protocols and social distancing. This issue is of paramount importance in dentistry clinics.

Objectives: The study aimed to evaluate the knowledge, attitude, and practice of dentists in Ahvaz regarding COVID-19.

Methods: The study population encompassed all dentists who were the WhatsApp group members of the Iranian Dental Association in Ahvaz, Iran. The online questionnaire link was shared on the social network on October 1, and the member dentists were asked to answer the questionnaire addressing demographic characteristics, knowledge about the signs and symptoms of COVID-19 disease, virus transmission method, and infection control method. These items assessed the dentists' attitude and practice in the treatment procedure of the COVID-19 patients.

Results: Out of 227 group members, 171 dentists observed the questionnaire link, and 108 persons answered the questions. Most of the respondents were $40-49$ years old and male. Moreover, $19 \%$ of the participants had dentistry specialization, $60 \%$ of the dentists had received no training on infection control in dentistry, 66\% of these individuals had passed the COVID-19 training course, and $23 \%$ of the dentists believed that the COVID-19 incubation period was 7-14 days. Most of the dentists were aware of the symptoms of COVID-19 and the diagnosis techniques for patients with COVID-19. They correctly reported the disease transmission and the disease prevention methods in the dental clinic. The dentists acted differently in using personal equipment, and only $75 \%$ of these individuals used the N95 Mask during the treatment of patients. About 13\% of the dentists did not study the protocol proposed by the Ministry of Health for reopening the dental clinic during the COVID-19 outbreak period.

Conclusions: The Ahvazian dentists are aware of the symptoms of COVID-19, its transmission, and the infection control protocol in dental clinics. However, they did not fully observe the protocols on wearing personal protective equipment for themselves and clinic staff; hence, barriers to using personal protective equipment should be eliminated, and the implementation of the protocols in dental clinics should be monitored more strictly.
\end{abstract}

Keywords: Protection, Protocol, Dentist, COVID-19

\section{Background}

The COVID-19 disease is a highly contagious viral disease first noticed in Wuhan, China, and led to the outbreak of pneumonia in other regions of the world. The World Health Organization declared this disease a public health emergency in January $2020(1,2)$ and a pandemic in March 2020 (3). According to the WHO report, 574856 definitive cases of COVID-19 infection and 32953 deaths caused by COVID-19 were reported in Iran by October 27, 2020 (4). The statistics on individuals with COVID-19 is increasing quickly as the disease is easily transmitted through droplets and aerosols emerge during talking, coughing, and sneezing directly or indirectly among those not observing social distancing with keeping close contact with each other ( $<6$ feet). Asymptomatic patients play a critical role in this transmission process. Unfortunately, medical staff, including dentists, are more at risk for COVID-19 than other individuals in society. In the dental office environment, aerosols are produced because of rotating devices and water spray, and these aerosols then combine with saliva and blood in patients' mouths, resulting in high contamination with bacteria, fungi, and viruses. These aerosols stay suspended in the office air for a long period 
or deposit on surfaces, thereby causing pathogenesis. In other words, dentists are directly or indirectly exposed to these infectious particles, and there is an increased risk of COVID-19 transmission in dental offices (1, 2, 5-11). Accordingly, designing effective infection control programs is vital to prevent the spread of the COVID-19 virus (8).

The Ministry of Health of the Islamic Republic of Iran issued guidelines on the operation of dental offices during the outbreak, which include strategies to reduce the transmission risk of the COVID-19 infection in the dental environment, and dentists are required to comply with them. Similar to international guidelines in this field, the guidelines address the use of personal protective equipment by dentists, office staff, and patients, disinfection and sterilization requirement sand methods in office environment and equipment, proper office ventilation, screening patients for COVID-19, patient appointment, social distancing, and educational brochures for patients in offices (2, 610).

The critical point in all the guidelines is that all clients have to be considered as infected, the infection control protocol should be observed equally for all patients, and treatments should be limited to emergency ones. Relevant knowledge of a topic can affect a person's attitude and performance. Cagetti et al. in Italy reported the dentists' awareness of COVID-19 in Milan, where the infection rate was higher compared to other Italian cities, to be about $71 \%$ (12). Duruk et al. concluded that only $68 \%$ of Turkey dentists were aware of COVID-19, and only $12 \%$ wore N95 Masks during the treatment procedures (10). In a previous study using a questionnaire, it was revealed that dentists' awareness and compliance with infection control during the treatment procedures of patients with viral infections were not highly satisfactory (13-16). Moreover, dental centers with many patients or centers offering cheap dental treatment services are unwilling to implement preventive recommendations on infection control $(17,18)$. Non-compliance with the infection control principles, especially regarding COVID-19, is frequently observed in Iran like many countries.

\section{Objectives}

Implementing infection control principles is essential, and increasing dentists' awareness is of paramount importance to promote infection control methods further. Accordingly, the present study was to evaluate the Ahvazian dentists' awareness, understanding, and attitudes towards COVID-19 and detect the need to control the viral infection.

\section{Methods}

This cross-sectional study was conducted in the Oral Diseases Department of the Ahvaz jundishapur University of Medical Sciences. The statistical population encompassed all 227 Ahvazian dentist who were the WhatsApp group members of the Ahvaz General Dentists Association (227 group members out of about 507 Ahvazian dentists) to validate the content of the developed questions. This group had been created by the Ahvaz General Dentists Association, and only dentists working in Ahvaz were allowed for membership after obtaining approval from the relevant association. This WhatsApp group had a history of 10 years, via which all dentists were getting informed about the scientific and professional news on dentistry. Accordingly, the dentists who were the members of this group participated in this research. The members of the group ( $n=227$ (were asked for cooperation. Before sharing the questionnaire link in the group, their medical ID No. was received to ensure that they were dentists. To observe information confidentiality, the respondents were not asked to record their detailed personal information. Inclusion criteria were being a dentist and familiarity with the Persian language, and the exclusion criterion was unwillingness to participate in the study. The participants filled in the questionnaires anonymously, and no personal information was collected. The study was approved by the Ethics Committee of the Ahvaz Jundishapur University of Medical Sciences (Code: IR.AJUMS.REC.1399.427) and observed the principles outlined in the Declaration of Helsinki for human investigations. After reviewing relevant articles and international guidelines, the authors, who were members of the Iranian Association of Oral medicine, developed the questionnaire $(10,12,15,19,20)$. The questionnaire was prepared in Persian and included questions on demographic characteristics, dentists' knowledge, their attitudes and perceptions of COVID-19, and their practice of infection control in clinics. The questionnaire's questions were in the multiple-choice form and were divided into several sections: dentists' demographic characteristics, dentists' knowledge of the incubation period, symptoms, the transmission methods of COVID-19, the COVID-19 infection control techniques, and dentists' attitudes towards treating patients with COVID-19.

The questions and their objectives were submitted to five oral and maxillofacial specialists to confirm their validity in evaluating awareness. They were asked to score each question based on its coordination with the objectives (one for highly coordinated questions and three for low-coordinated questions). Then the questions rated two or three by the majority of experts were removed from the questionnaire. The experts confirmed its formal validity as 
well. Cronbach's coefficient for 15 dentists was 0.75 after eliminating the questions decreasing reliability in evaluating the reliability of the questionnaire.

This study started on 2020.09.22 and ended on 2020.10.06. At the beginning of the study, 3712 cases of COVID-19 infection and 175 deaths caused by the COVID-19 infection were reported. The research data were collected using an online questionnaire (https://porsline.ir/ website), and the link was sent to the participants. The collected were analyzed using SPSS software version 21. Descriptive analysis was used to describe the study items. Mean and standard deviation were used to describe the continuous variables, and the percentage was used for the categorical data.

\section{Results}

\subsection{Characteristics of Participants}

One hundred and eight dentists (45 females and 63 males) answered the questions, indicating an approximate participation rate of $63 \%$ (108 dentists out of 171 dentists who read the message). The participants in the study were 40-49 years old (34.3\%), who were mainly working in private clinics $(n=84)$, and $62 \%$ of the participants were active. Further, $81 \%$ of the participants were general dentists, and $19 \%$ had a dental specialty. Table 1 presents the participants' demographic characteristics. Almost $60 \%$ of the dentists had received no training on the principles of dental infection control, and 66\% did not take the COVID-19 training course. Thirty-four participants (31.5\%) had a person with COVID-19 in their family. The mean response time to the questionnaire by dentists was about nine minutes.

\subsection{Knowledge of COVID-19 Incubation Period, Symptoms, and Transmission Methods}

In this study, 69 (63.9\%), 25 (23.1\%), 8 (7.4\%), and 6 (5.6\%) dentists answered the questionnaire during days 1-14, 7-14, 2-7, and 7-21, respectively. In response to the incubation period of the COVID-19 disease, 9 (63.9\%) dentists considered 1-14 days, and 25 (23.1\%) dentists answered 7-14 days, 8 (7.4\%) dentists assumed 2-7 days, and 6 (5.6\%) persons reported 7-21 days. Table 2 presents the dentists' responses to the COVID-19 symptoms. A majority of dentists reported fever $(n=105,97.2 \%)$ and shortness of breath $(n=105,97.2 \%)$, dry $\operatorname{cough}(n=103,95.4 \%)$, and the decreased sense of smell $(n=$ $101,93.5 \%)$ as the symptoms of the COVID-19 infection. Further, the decreased sense of taste $(n=94.87 \%)$, headache ( $n$ $=79,73.1 \%)$, color change in tongue and oral mucosa $(n=22$, $20.4 \%$ ), and decreased saliva $(n=16,14.8 \%$ ) were the other symptoms in this regard. The probability of asymptomatic

\begin{tabular}{ll}
\hline Table 1. Demographic Characteristics of Dentists & No, $\%$ \\
\hline Variables & \\
\hline Gender & 45,42 \\
\hline Female & 63,58 \\
\hline Male & \\
\hline Age (y) & $15,13.9$ \\
\hline$<30$ & $93,86.1$ \\
\hline 30 or > 30 & \\
\hline Place of activity & $84,77.8$ \\
\hline Private office & $18,16.7$ \\
\hline State clinic & $24,22.2$ \\
\hline Private clinic & $1,0.9$ \\
\hline Charity clinic & $1,0.9$ \\
\hline Military clinic & $87,80.6$ \\
\hline Level of education & $21,19.4$ \\
\hline General dentist & \\
\hline Specialist dentist & \\
\hline
\end{tabular}

COVID-19 was also reported to be $77.8 \%(n=84)$. When dentists were asked about the symptoms to be considered in diagnosing patients suspected of COVID-19, the presence of respiratory symptoms in the last two weeks was reported in 103 cases (95.4\%), history of contact with suspected patients during the last two weeks was reported in 104 cases (96.3\%), and travel to places with the highest disease prevalence during the last two weeks was reported in 100 cases (92.6\%). Moreover, most dentists provided correct answers regarding the COVID-19 transmission methods.

\subsection{Knowledge About COVID-19 Transmission Methods in Den- tal Settings}

Most of the dentists reported that frequent handwashing with soap and water or the use of alcoholic solutions ( $n=105,97.2 \%)$, wearing personal protective equipment by clinic staff $(n=102,95.4 \%)$, regular cleaning and disinfection of surfaces ( $\mathrm{n}=104,96.3 \%$ ) should be considered as techniques to prevent the COVID-19 transmission. Almost all dentists reported that regular replacement of gloves (n $=107,99.1 \%)$ and masks $(n=97,89.8)$ is essential in preventing the COVID-19 transmission to other patients and dentists.

\subsection{Perception of COVID-19}

In this study, 103 (95.4\%) dentists considered COVID-19 disease as a dangerous and extremely dangerous disease, four (3.7\%) dentists considered it to be moderately dangerous, one person (0.9) considered it safe, and 106 (98.2\%) in- 


\begin{tabular}{|cc}
\hline Table 2. Dentists' Awareness of COVID-19 Infection & No, $\%$ \\
\hline Variable & \\
\hline Incubation period (day) & $69,63.9$ \\
\hline $1-14$ & $8,7.4$ \\
\hline $2-7$ & $25,23.1$ \\
\hline $7-14$ & $6,5.6$ \\
\hline $7-21$ & \\
\hline Symptoms & $105,97.2$ \\
\hline Fever & $103,95.4$ \\
\hline Dry coughs & $105,97.2$ \\
\hline Shortness of breath & $53,49.1$ \\
\hline Skin rash & $89,82.4$ \\
\hline Diarrhea & $101,93.5$ \\
\hline Decreased sense of smell & 94,87 \\
\hline Decreased sense of taste & $16,14.8$ \\
\hline Saliva reduction & $79,73.1$ \\
\hline Headache & 22,20 \\
\hline Problems such as tongue and oral mucosa discoloration & $84,77.8$ \\
\hline Probablyasymptomatic & $2,1.9$ \\
\hline I do not know. & \\
\hline Transfer Method & $107,99.1$ \\
\hline Coughing and sneezing & $106,98.1$ \\
\hline Shaking hands and kissing & $99,91.7$ \\
\hline Touching handles and tables & $42,38.9$ \\
\hline Eating and drinking & \\
\hline
\end{tabular}

dividuals reported it a a serious challenge to the health system.

\subsection{Attitudes and Practices of Dentists on COVID-19}

About $88.9 \%$ of the dentists $(n=96)$ preferred to refrain from providing dental treatment for patients with COVID19 , and $87 \%(n=94)$ of the dentists sent patients with cough and sneezing visiting their clinic to the hospital with no treatment, and $13 \%(n=14)$ of the participants referred the patient to the hospital after treatment. Furthermore, 106 (98.1\%) dentists believed that patients should sit down away from each other, wear masks in waiting rooms, and wash their hands before sitting on the unit. Moreover, 105 (97.2\%) dentists also believed that if clinic staffs have flulike symptoms, they are not allowed to be present in the clinic, and $8.3 \%(\mathrm{n}=9)$ of the dentists did not know what to do if they had the COVID-19signs and symptoms. Furthermore, 93 (86.9\%) dentists had studied the protocol proposed by the Ministry of Health to reopen a dental clinic during the COVID-19 outbreak. The dentists' practice of the basics of infection control is presented in Table 3.

\begin{tabular}{|c|c|}
\hline Variable & No, $\%$ \\
\hline $\begin{array}{l}\text { Before contacting any patient, I observe hand hygiene, } \\
\text { including proper handwashing with soap and water or } \\
\text { hand disinfection with alcohol-based solutions. }\end{array}$ & $97,89.8$ \\
\hline $\begin{array}{l}\text { I have learned the necessary points about COVID 19, and I ask } \\
\text { her (him) to follow it. }\end{array}$ & $100,92.6$ \\
\hline $\begin{array}{l}\text { I wear an N95, N99, or similar for the patient before and } \\
\text { during dental treatment procedure. }\end{array}$ & $82,75.9$ \\
\hline $\begin{array}{l}\text { I wear a regular mask for the patient before and during } \\
\text { dental treatment procedure. }\end{array}$ & $46,42.6$ \\
\hline $\begin{array}{l}\text { I wear eye protection for the patient before and during } \\
\text { dental treatment procedure. }\end{array}$ & $86,79.6$ \\
\hline $\begin{array}{l}\text { I wear face protection for the patient before and during } \\
\text { dental treatment procedure. }\end{array}$ & $78,72.2$ \\
\hline $\begin{array}{l}\text { I wear a hat for the patient before and during dental } \\
\text { treatment procedure. }\end{array}$ & $65,60.2$ \\
\hline My assistant uses eye protection. & $59,54.6$ \\
\hline My assistant uses an N95, N99, or similar. & $44,40.7$ \\
\hline $\begin{array}{l}\text { I refrain from eating and drinking in the examination, } \\
\text { treatment, and waiting rooms. }\end{array}$ & $61,56.5$ \\
\hline $\begin{array}{l}\text { The surface and back cover of the unit are replaced before } \\
\text { the next patient enters the room. }\end{array}$ & $60,55.6$ \\
\hline
\end{tabular}

\section{Discussion}

The coronavirus epidemic (COVID-19) is an emergency and a public health concern. All individuals, including dentists, are at risk for COVID-19 infection during this epidemic (21). Our results showed that a majority of the dentists in Ahvaz were aware of the COVID-19 symptoms, its transmission methods, and the infection control protocol in dental clinics; however, they had no appropriate practice of the aforementioned points. This finding is similar to those reported by Nasser et al. (21) in Lebanon, suggesting that a majority of the Lebanese dentists had satisfactory knowledge, while approximately half of the dentists had poor practice.

The present finding is also similar to a multinational study by Kamate et al. (22) and studies by Khader et al. in Jordan (15), studies by Saqlain et al. in Pakistan (23), and another study in Iran by Sarkarat (24). All these studies indicated that a relatively high proportion of dentists had appropriate knowledge of the coronavirus protection techniques. However, as the questions became more techni$\mathrm{cal}$, the percentage of correct answers decreased (24). Although the dentists had high levels of knowledge for some questions, their awareness of some items was not satisfactory. These findings are comparable to other studies. 
Dentists in our study had full knowledge of the COVID19 symptoms, which is similar to findings reported by Khadir (15) and Sarkarat (24) on Jordanian and Iranian dentists' knowledge of COVID-1 and in contrast with those reported by Baser (25), and Abolfotouh (26). In the present study, $89.8 \%$ of the dentists were aware of handwashing; however, in another study in Iran by Sarkarat (24), the responses to two questions on handwashing were not satisfactory. On the other hand, in our study, the dentists had different levels of knowledge about the COVID19incubation period. The COVID-19 incubation period is estimated to vary from 5-6 days to 14 days on average (27). Our findings showed that only $23.1 \%$ of the dentists reported 7-14 days in this regard. This is similar to the findings of Khader's study in Jordan (15). In this regard, inconsistencies in the findings of studies on awareness may be due to dentists' lack of up-to-date information. Regarding the findings of this study and similar studies, dentists are obliged to follow preventive measures for all patients at all times (14).

The pandemic nature of COVID-19, many media and policymakers' attention to this issue, and public education programs held via mass media by officials have increased the knowledge of all community members, including dentists, about the COVID-19 symptoms (28). Due to the critical nature of this disease and given the findings of this study and similar studies, dentists should follow and update their information about different aspects of COVID19. Dentists' knowledge of the main COVID-19 symptoms helps them identify threats and adopt appropriate measures in this regard, and this would play an essential role in controlling the spread of this disease (14). Individuals' awareness about the COVID-19 incubation period is critical since it helps us determine the safe period for providing dental treatment to suspected patients. Baseer and Abolfotouh showed that dentists are less aware of the symptoms of infectious respiratory diseases compared to other health professionals; however, they need close contact and communication with patients to provide dental treatment $(25,26)$. In this regard, knowledge about handwashing is extremely important in controlling diseases such as MERS or COVID-19 and needs to be promoted (25).

Regarding the dentists' perception of the COVID-19 disease, the findings showed that above $95 \%$ of the dentists considered the COVID19 disease to be highly dangerous and dangerous, and above $98 \%$ of these individuals considered it as a serious health problem. Furthermore, dentists' perception of the dangers of this disease made about $90 \%$ of dentists be unwilling to provide treatment for the COVID-19 patients, and a majority of the dentists (98.1\%) asked their patients to wear masks in the waiting room and observe social distancing. However, in Khadir's study, 71\% of the dentists considered COVID-19 a moderate risk, and about $21 \%$ did not consider it necessary for patients to sit down away from each other and wear a mask in the waiting room (15). This inconsistency of the findings might be due to differences in prevalence and mortality rates in Iran (300 persons per day on average) and Jordan (one person per day on average) $(4,15)$

Although this study showed relatively high levels of knowledge, perception, and attitude towards COVID-19, the practice level was not satisfactory in some cases. For example, most of the dentists in our study considered frequent hand washing and the use of personal protective equipment as methods to prevent the COVID-19 infection transmission; however, in practice, $75.9 \%$ of the dentist and $40.7 \%$ of their assistants wore N95 or N99 Masks or similar, $79.6 \%$ of the dentists and $54.6 \%$ of their assistants wore eye protection equipment, and $72.2 \%$ of the dentists wore face protection before and during dental treatment procedures for the patient. These findings are consistent with those of a study in Lebanon, which revealed about half of the respondents (58.7\%) followed precautionary measures (21). Since appropriate knowledge of a disease can lead to more positive attitudes and more effective protection and prevention practices, and given that acceptable knowledge is essential (22), the present research, consistent with some other studies $(15,22)$, showed that the association among knowledge, attitude, and practice cannot be understood simply and other factors such as professional ethics, work experience, updated information sources of COVID-19, the access to or the lack of access to personal protective equipment, and others should be considered.

In our study, $86.9 \%$ of the dentists had studied the protocol issued by the Ministry of Health to reopen a dental clinic during the COVID-19 outbreak. In Lebanon (21), 73.37\% of dentists used official government websites such as the WHO website as the main source of information. Not studying the protocol proposed by the Ministry of Health by several dentists in this study (13.1\%) can justify the dentists' different behaviors in dealing with suspected staffs or patients. For example, the dentists in this study had different attitudes towards patients who were coughing and sneezing. About $22.2 \%$ of the dentists did not visit the patients; however, $64.8 \%$ of the dentists without providing any treatment referred the patient to hospital after admission, and $13 \%$ of the dentists referred patients to the hospital after treatment. A few dentists (2.8\%) were allowed to work in clinics based on the protocol of the Ministry of Health of Iran. Moreover, the access or lack of access to equipment can affect dentists' practice. This finding was confirmed by Tysiac-mista and Dziedzic in Netherlands, concluding that the access to or the lack of access to personal protective equipment plays a major role in den- 
tists' decision to pursue their work during the coronavirus pandemic (29).

In this study, the number of males was more than the females. The heterogeneity might be attributed to the fact that there are more male dentists in Iran and Ahvaz. Although there are no reliable statistics in this regard, studies on the dentists' community have also confirmed the number of male dentists was more than that of female dentists $(24,30,31)$

The present study had some limitations, including small sample size and limited samples in Khuzestan province. Moreover, only those who had access to the WhatsApp social network during the short data collection period had a chance to participate in the study, and they evidently had access to the COVID-19 information on this social network. However, all the dentists who had access to this social media did not complete the questionnaire, and the participation rate was $63 \%$. These factors might have resulted in some bias in the results; hence, the findings cannot be generalized to all dentists.

\subsection{Conclusions}

The Ahvazian dentists are aware of the symptoms of COVID-19, its transmission, and the infection control protocol in dental clinics. However, they did not fully observed the protocols on wearing personal protective equipment for themselves and clinic staff; hence, barriers to using personal protective equipment should be eliminated, and the implementation of the protocols in dental clinics should be monitored more strictly.

\section{Acknowledgments}

The study was extracted from a research project funded by the Ahvaz Jundishapur University of Medical Sciences. Hereby, the authors appreciate the project sponsor and the dentists participating in the project.

\section{Footnotes}

Authors' Contribution: Study concept and design: M.Sh.; Study concept and design: B.M; M.Sh; contributed to the development of the protocol, abstracted data, and prepared the manuscript: S.M.M; participated in designing the evaluation: E.GH.

Conflict of Interests: The authors declare no relevant financial interests in the material in this manuscript.

Ethical Approval: The study was approved by the Ethics Committee of the Ahvaz Jundishapur University of Medical Sciences (Code: IR.AJUMS.REC.1399.427) and observed the principles outlined in the Declaration of Helsinki for human investigations.
Funding/Support: The research budget was provided by the Ahvaz Jundishapur University of Medical Sciences.

\section{References}

1. Gambhir RS, Dhaliwal JS, Aggarwal A, Anand S, Anand V, Bhangu AK. Covid-19: a survey on knowledge, awareness and hygiene practices among dental health professionals in an Indian scenario. Rocz Panstw Zakl Hig. 2020;71(2):223-9. doi: 10.32394/rpzh.2020.0115.

2. Abdul Rehman M, Mariam Q, Abduljabbar T, Vohra F, Shakoor M, Ahmed N. Knowledge, Awareness and Practice of Health care Professionals amid SARS-CoV-2, Corona Virus Disease Outbreak. PakJMed Sci. 2020;36(COVID19-S4). doi: 10.12669/pjms.36.COVID19-S4.2704.

3. De Stefani A, Bruno G, Mutinelli S, Gracco A. COVID-19 Outbreak Perception in Italian Dentists. Int J Environ Res Public Health. 2020;17(11):3867. doi: 10.3390/ijerph17113867.

4. World Health Organization. Iran (Islamic Republic of) Situation. New York: World Health Organization; 2021, [cited October 16]. Available from: https://COVID19.who.int/region/emro/country/ir.

5. Peditto M, Scapellato S, Marcianò A, Costa P, Oteri G. Dentistry during the COVID-19 Epidemic: An Italian Workflow for the Management of Dental Practice. Int J Environ Res Public Health. 2020;17(9). doi: 10.3390/ijerph17093325. [PubMed: 32403248].

6. Dar-Odeh N, Elsayed S, Babkair H, Abu-Hammad S, Althagafi N, Bahabri $\mathrm{R}$, et al. What the dental practitioner needs to know about pharmaco-therapeutic modalities of COVID-19 treatment: A review. J Dent Sci. 2021;16(3):806-16. doi: 10.1016/j.jds.2020.11.007. [PubMed: 33230404]. [PubMed Central: PMCPmc7674127].

7. Quadri MFA, Jafer MA, Alqahtani AS, Al Mutahar SAB, Odabi NI, Daghriri AA, et al. Novel corona virus disease (COVID-19) awareness among the dental interns, dental auxiliaries and dental specialists in Saudi Arabia: A nationwide study.JInfect Public Health. 2020;13(6):85664. doi: 10.1016/j.jiph.2020.05.010. [PubMed:32475807]. [PubMed Central: PMCPmc7255993]

8. Peng X, Xu X, Li Y, Cheng L, Zhou X, Ren B. Transmission routes of 2019$\mathrm{nCoV}$ and controls in dental practice. Int J Oral Sci. 2020;12(1):9. doi: 10.1038/s41368-020-0075-9. [PubMed: 32127517].

9. Lo Giudice R. The Severe Acute Respiratory Syndrome Coronavirus2 (SARS CoV-2) in Dentistry. Management of Biological Risk in Dental Practice. Int J Environ Res Public Health. 2020;17(9). doi: 10.3390/ijerph17093067. [PubMed: 32354081]. [PubMed Central: PMCPmc7246879].

10. Duruk G, Gümüşboğa ZŞ, Çolak C. Investigation of Turkish dentists' clinical attitudes and behaviors towards the COVID-19 pandemic: a survey study. Braz Oral Res. 2020;34. e054. doi: 10.1590/1807-3107bor2020.vol34.0054. [PubMed: 32490887].

11. Baghizadeh Fini M. What dentists need to know about COVID-19. Oral Oncol. 2020;105:104741. doi: 10.1016/j.oraloncology.2020.104741. [PubMed: 32380453]. [PubMed Central: PMCPmc7186204].

12. Cagetti MG, Cairoli JL, Senna A, Campus G. COVID-19 Outbreak in North Italy: An Overview on Dentistry. A Questionnaire Survey. Int J Environ Res Public Health.2020;17(11). doi:10.3390/ijerph17113835. [PubMed: 32481672]. [PubMed Central: PMCPmc7312000].

13. Khosravanifard B, Rakhshan V, Najafi-Salehi L, Sherafat S. Tehran dentists' knowledge and attitudes towards hepatitis B and their willingness to treat simulated hepatitis B positive patients. East Mediterr Health J. 2014;20(8):498-507. [PubMed: 25150357].

14. Gaffar BO, El Tantawi M, Al-Ansari AA, AlAgl AS, Farooqi FA, Almas KM Knowledge and practices of dentists regarding MERS-CoV. A crosssectional survey in Saudi Arabia. Saudi Med J. 2019;40(7):714-20. doi: 10.15537/smj.2019.7.24304. [PubMed: 31287133]. [PubMed Central: PMCPmc6757212]. 
15. Khader Y, Al Nsour M, Al-Batayneh OB, Saadeh R, Bashier H, Alfaqih $\mathrm{M}$, et al. Dentists' Awareness, Perception, and Attitude Regarding COVID-19 and Infection Control: Cross-Sectional Study Among Jordanian Dentists. JMIR Public Health Surveill. 2020;6(2). e18798. doi: 10.2196/18798.

16. Brug J, Aro AR, Oenema A, de Zwart O, Richardus JH, Bishop GD. SARS risk perception, knowledge, precautions, and information sources, the Netherlands. Emerg Infect Dis. 2004;10(8):1486-9. doi: 10.3201/eid1008.040283. [PubMed: 15496256]. [PubMed Central: PMCPmc3320399].

17. Matsuda JK, Grinbaum RS, Davidowicz H. The assessment of infection control in dental practices in the municipality of São Paulo. Braz J Infect Dis. 2011;15(1):45-51. [PubMed: 21412589].

18. Mehtar S, Shisana O, Mosala T, Dunbar R. Infection control practices in public dental care services: findings from one South African Province. J Hosp Infect. 2007;66(1):65-70. doi: 10.1016/j.jhin.2007.02.008. [PubMed: 17433494].

19. Meng L, Hua F, Bian Z. Coronavirus Disease 2019 (COVID-19): Emerging and Future Challenges for Dental and Oral Medicine. J Dent Res. 2020;99(5):481-7. doi: 10.1177/0022034520914246.

20. World Health Organization. Clinical management of severe acute respiratory infection when novel coronavirus (2019-nCoV) infection is suspected: interim guidance. 2020.21 p.

21. Nasser Z, Fares Y, Daoud R, Abou-Abbas L. Assessment of knowledge and practice of dentists towards Coronavirus Disease (COVID-19): a cross-sectional survey from Lebanon. BMC Oral Health. 2020;20(1):281 doi: 10.1186/s12903-020-01273-6. [PubMed: 33050914]. [PubMed Central: PMCPmc7552581].

22. Kamate SK, Sharma S, Thakar S, Srivastava D, Sengupta K, Hadi AJ, et al. Assessing Knowledge, Attitudes and Practices of dental practitioners regarding the COVID-19 pandemic: A multinational study. Dent Med Probl. 2020;57(1):11-7. doi:10.17219/dmp/119743. [PubMed: 32307930].

23. Saqlain M, Munir MM, Rehman SU, Gulzar A, Naz S, Ahmed Z, et al. Knowledge, attitude, practice and perceived barriers among healthcare workers regarding COVID-19: a cross-sectional survey from Pak- istan. J Hosp Infect. 2020;105(3):419-23. doi: 10.1016/j.jhin.2020.05.007. [PubMed: 32437822]. [PubMed Central: PMCPmc7211584].

24. Sarkarat F, Tootoonchian A, Hosseinpour M, Moghadasi M, Rakhshan V. Knowledge of Iranian Dentists, Dental Specialists, and Dental Students Towards COVID-19: A Preliminary Survey of 778 Subjects. Shiraz E-Med J. 2020;21(12).

25. Baseer MA, Ahlawat J, Ansari SH, Alakras AR, Mahrous R, Alenazi AM. Awareness of droplet and airborne isolation precautions among dental health professionals during the outbreak of corona virus infection in Riyadh city, Saudi Arabia. J Clin Exp Dent. 2016;1;8(4):e379-87. doi: 10.4317/jced.52811.

26. Abolfotouh MA, AlQarni AA, Al-Ghamdi SM, Salam M, Al-Assiri MH, Balkhy HH. An assessment of the level of concern among hospitalbased health-care workers regarding MERS outbreaks in Saudi Arabia. BMC Infect Dis. 2017;17(1):4. doi: 10.1186/s12879-016-2096-8.

27. Backer JA, Klinkenberg D, Wallinga J. Incubation period of 2019 novel coronavirus (2019-nCoV) infections among travellers from Wuhan, China, 20-28 January 2020. Euro Surveill. 2020;25(5). doi:10.2807/15607917.es.2020.25.5.2000062. [PubMed: 32046819]. [PubMed Central: PMCPmc7014672].

28. Nourmoradi H, Kazembeigi F, Kakaei H, Jalilian M, Mirzaei A. Assessment of knowledge, attitude, and practice toward covid-19 among a sample of iranian general population. Open Access Maced J Med Sci. 2020;8(T1):167-74

29. Tysiac-Miśta M, Dziedzic A. The Attitudes and Professional Approaches of Dental Practitioners during the COVID-19 Outbreak in Poland: A Cross-Sectional Survey. Int J Environ Res Public Health. 2020;17(13). doi: 10.3390/ijerph17134703. [PubMed: 32629915].

30. Shooriabi M, Hojjat SM, Satvati SAR, Sharifi R. Evaluating the Knowledge and Performance of Dentists about Halitosis in Ahvaz, Tehran and Gorgan during 2014-2015. Int J Med Res Health Sci. 2016;5(9):167-73.

31. Ghasemi H, Mirdehghan SR, Namdari M, Bayat F. Offspring sex ratio of Iranian dentists. Environ Health Prev Med. 2016;21(6):446-9. doi: 10.1007/s12199-016-0546-y. [PubMed: 27329277]. [PubMed Central: PMCPmc5112190]. 